\title{
Parent-reported sleep problems in children and adolescents with sickle cell disease: relationship to health-related quality of life
}

\author{
Serkan Gunes ${ }^{1}$, Rahime Aldemir ${ }^{1}$, Adem Gunes ${ }^{1}$, Ozalp Ekinci ${ }^{2}$
}

\begin{abstract}
${ }^{1}$ Department of Child and Adolescent Psychiatry, Hatay State Hospital, Hatay, Turkey ${ }^{2}$ Department of Child and Adolescent Psychiatry, Medical Faculty, University of Health Sciences, Istanbul, Turkey
\end{abstract}

Submitted: 13 March 2020; Accepted: 18 June 2020

Online publication: 26 January 2021

Arch Med Sci 2022; 18 (3): 659-665

DOI: https://doi.org/10.5114/aoms/124154

Copyright (c) 2021 Termedia \& Banach

\section{Abstract}

Introduction: Children with sickle cell disease (SCD) can present a variety of clinical symptoms that may affect their sleep and health-related quality of life (HRQOL). This study aims to investigate the relation between sleep problems and HRQOL in children and adolescents with SCD.

Material and methods: The sample included 86 children and adolescents in the SCD patient group and 82 healthy controls, with an age range of 8-16years. Subjects for the study were recruited from the Sickle Cell and Thalassemia Center of Hatay State Hospital, Hatay, Turkey. The Children's Sleep Habits Questionnaire (CSHQ) was used to evaluate sleep problems and Kinder Lebensqualitätsfragebogen: Children's Quality of Life Questionnaire - revised (KINDL-R) was used to examine HRQOL.

Results: Total score, bedtime resistance, and night waking subscores of CSHQ were significantly higher in children with SCD when compared to healthy children. Total score, physical well-being, emotional well-being, social, and school subscores of KINDL-R were significantly lower in the patient group. Among SCD children, total score, bedtime resistance, sleep onset delay, daytime sleepiness, and parasomnias subscores of CSHQ were negatively correlated with KINDL-R total score. In the regression model, disease severity and CSHQ total score had significant negative associations with KINDL-R total score.

Conclusions: Sleep problems in SCD children appear to be negatively linked with HRQOL. Disease severity and sleep problems may be predictors of overall HRQOL in children and adolescents with SCD.

Key words: anemia, hemoglobinopathies, quality of life, sleep.

\section{Introduction}

Sickle cell disease (SCD) is a class of chronic, recessively inherited red blood diseases characterized by the existence of abnormal hemoglobin [1]. Sickle cell disease is a significant global health problem affecting about 400,000 newborns each year [2]. The clinical presentations of SCD are different and include acute and chronic symptoms. Acute clinical symptoms are painful vaso-occlusive episodes, and hematological and infectious crisis. In the chronic period, SCD may affect many organs and systems [3]. Since SCD is a life-long disorder, it may also result in a range of cognitive and psychosocial complications [1].

\author{
Corresponding author: \\ Serkan Gunes \\ Department of Child \\ and Adolescent Psychiatry \\ Hatay State Hospital \\ Güzelburç \\ 31001 Antakya/Hatay, Turkey \\ E-mail: \\ dr_sgunes@hotmail.com
}


Sleep is a crucial factor in the development of children. Disturbed sleep has been related to impairments in attention, memory, behaviors, school success, emotional status, and daily activities [4]. Pain-related sleep disorders are an important concern in SCD children and adolescents due to the high prevalence of acute and chronic pain [5]. As a bidirectional theory of pain and sleep disorder, pain may not simply affect sleep, but poor sleep quality may also have unfavorable impacts on pain handling [6]. Because of this relationship, a better understanding of sleep may improve pain management and health-related quality of life in children and adolescents with SCD [7].

Health-related quality of life (HRQOL) is a measure of physical, emotional, and social well-being which is influenced by medical conditions, treatments, and interventions [8, 9]. Many clinical symptoms and complications may affect HRQOL in SCD children. Recurrent pain episodes, developmental delays, anemia, low exercise tolerance, and end-organ complications can accompany SCD. In this context, HRQOL is a significant construct in children and adolescents with SCD to define the health profile and functional status, to evaluate the effects of medical treatments, and to assess children's needs [10].

Although there are studies about sleep problems and HRQOL in children with SCD, to our knowledge, this is the first study examining the relationship between sleep problems and HRQOL. In the present study, our aim is to compare sleep disturbances and HRQOL in children and adolescents with SCD and healthy participants and to investigate the relationship between sleep disturbances and HRQOL. We hypothesized that sleep problems might be more common in children with SCD than in healthy children and sleep problems might be associated with overall HRQOL in SCD children.

\section{Material and methods}

This was a cross-sectional study that included SCD children and adolescents who presented to the Sickle Cell and Thalassemia Center of Hatay State Hospital in Hatay in Turkey. The study period lasted from January to June 2019.

\section{Study groups}

The inclusion criteria of the patient group were: 1) within the age range of 8-16 years, 2) not presenting a physical disease other than SCD, 3) a diagnosis of SCD for 2-4 years, 4) normal intellectual capacity based on a Wechsler Intelligence Scale for Children-Revised (WISC-R) [11] IQ score higher than 80.

For the control group, the following inclusion criteria were used: 1) within the age range of 8-16 years, 2) not presenting a physical disease or a psychiatric disorder according to DSM-5, 3) normal intellectual capacity based on a WISC-R full-scale IQ score higher than 80 .

For both the patient and control groups, children whose parents had a chronic physical illness, intellectual disability, motor or visual handicaps were excluded from the study since these problems might affect the opinions of parents about their children.

Ninety-eight children with SCD diagnosis were initially recruited, but twelve children were excluded from the study for the following reasons: 4 parents did not give permission for children to be included in the study, 3 children had a WISC-R full-scale IQ score under 80 , and 5 children had other chronic medical illnesses. In total, 86 children and adolescents with SCD and 82 healthy individuals fulfilled the study conditions and participated in the study.

\section{Procedure}

Parents and their children with SCD were approached during their regular outpatient clinic appointments in the Sickle Cell and Thalassemia Center. After the appointment, they were referred to the Child and Adolescent Psychiatry Clinic of the same hospital by a trained nurse. At the clinic, an experienced psychologist performed the full-scale WISC-R in children and adolescents. After the full-scale WISC-R, a detailed psychiatric examination was performed by a child and adolescent psychiatry specialist and information regarding sociodemographics, disease type, and hospitalization was collected. The duration of the psychiatric examination was 45 min.

The control group included healthy children and adolescents without any physical and mental illness who were referred to the Child and Adolescent Psychiatry Clinic. These children included admissions for counseling purposes, e.g. sibling birth, school preference, friendship problems, and adaptation to adolescence. The full-scale WISC-R was also performed with these healthy children and adolescents by the same psychologist and a detailed psychiatric examination was also performed by the same child and adolescent psychiatry specialist.

The questionnaires were completed in a private, quiet room at the clinic by all parents of the participants.

\section{Measures \\ Children's Sleep Habits Questionnaire}

The Children's Sleep Habits Questionnaire (CSHQ) is a 45-item parent-report questionnaire assessing the sleep behaviors of children and 
adolescents [12, 13]. The CSHQ consists of the following parameters: sleep-disordered breathing; bedtime behavior and sleep onset; behavior occurring during sleep and night wakings; parasomnias; total sleep time; daytime sleepiness; and anxiety around sleep. The scale is filled retrospectively and parents are requested to remember their children's sleep patterns that occurred in the past week. Items are rated on a three-point scale: "usually" if the sleep behavior occurred five to seven times/week; "sometimes" for two to four times/week; and "rarely" for zero to one time/ week. Higher scores indicate more sleep disturbances [4]. The Turkish version of the scale was performed by Fiş et al. and used in this study [14].

\section{Kinder Lebensqualitätsfragebogen: \\ Children's Quality of Life Questionnaire - revised}

Kinder Lebensqualitätsfragebogen: Children's Quality of Life Questionnaire - revised (KINDL-R) is a measure to determine HRQOL in children and adolescents. The questionnaire examines HRQOL in six different dimensions: physical well-being, emotional well-being, self-worth, family-related well-being, friend-related well-being, and school-related well-being (daily functioning) [15]. Kinder Lebensqualitätsfragebogen: Children's Quality of Life Questionnaire - revised consists of 24 items referring to the past week with 5-point Likert-scales (from never to all the time), with 11 items being reverse-coded. The scores range from 0 to 100, and higher values show better HRQOL [8]. Eser et al. demonstrated the validity and reliability of the Turkish version of the questionnaire [16]. In this study, the KINDL-R parent questionnaire was used.

\section{Disease severity}

Disease severity was examined as a dual categorization of "mild" and "severe" according to the parameters used in previous studies $[1,17]$. The children and adolescents with a severe disease had end-organ damage, chronic transfusion therapy, twelve or more medical applications for pain in the last year, three or more hospitalizations required per year, based on medical records. The patients with a mild disease did not meet any of the above-mentioned parameters.

\section{Statistical analysis}

All statistics were performed using the Statistical Package for Social Sciences Software 21.0 (SPSS Inc., Chicago, IL, USA). Categorical data were analyzed using descriptive statistics and compared using the $\chi^{2}$ test. KolmogorovSmirnov and Shapiro-Wilk tests were performed to examine normality in continuous variables. Groups were compared using the independent samples $t$ test for normally distributed parametric variables $(95 \% \mathrm{Cl})$. Pearson's correlation analysis was performed to determine the relationships between CSHQ and KINDL-R scores. A hierarchical regression analysis was used to investigate whether demographic variables, disease severity, and sleep problems contribute to HRQOL of SCD children and adolescents. The demographic variables were entered in step 1 , disease severity in step 2, and all CSHQ scores were entered in step 3. A $p$-value of $<0.05$ was considered to indicate statistical significance.

\section{Ethical consideration}

The present study was performed according the principles of the Declaration of Helsinki (updated 2008) and the Ethics Committee of Adana City Hospital, Adana, Turkey, approved the study procedure. The approval number was 2019/566.

Before the administration, the nature and purpose of the study and the questionnaires were explained to all children and parents by a child and adolescent psychiatry specialist and written informed consent was obtained.

\section{Results}

The sample included 86 children and adolescents in the SCD patient group and 82 healthy controls, with an age range of 8-16 years (mean $\pm \mathrm{SD}=12.30 \pm 2.41)$. Mean age of the patient group was $12.17 \pm 2.36$ years, and $62.8 \%(n=54)$ were male. The control group had a mean age of $12.42 \pm 2.46$ years, and $59.8 \%(n=49)$ were male. SCD types of the patients were as follows: 80.2\% ( $n=69) \mathrm{HbSS}, 14.0 \%(n=12) \mathrm{HbSC}$, and $5.8 \%(n=5) \mathrm{HbS}$ b-thalassemia.

The comparison of CSHQ scores between the patient and control groups is presented in Table I. Children's Sleep Habits Questionnaire total score $(p=0.034)$, and bedtime resistance $(p=0.024)$ and night waking $(p=0.033)$ subscores were significantly higher in the patient group than the control group. Sleep onset delay, sleep anxiety, daytime sleepiness, parasomnias, and sleepdisordered breathing subscores were also higher in the patient group, but these differences were not significant $(p>0.05)$.

Table II shows the comparison of KINDL-R scores between the patient and control groups. Kinder Lebensqualitätsfragebogen: Children's Quality of Life Questionnaire - revised total score $(p=0.043)$, physical well-being ( $p=0.000)$, emotional wellbeing $(p=0.040)$, social $(p=0.002)$, and school $(p=0.021)$ subscores were significantly lower in the patient group than the control group. Self- 
Table I. Comparison of Children's Sleep Habits Questionnaire (CSHQ) scores between the patient and control groups

\begin{tabular}{|lcccc|}
\hline CSHQ & $\begin{array}{c}\text { Patients, } n=86 \\
\text { Mean (SD) }\end{array}$ & $\begin{array}{c}\text { Controls, } n=82 \\
\text { Mean (SD) }\end{array}$ & Cohen's $\boldsymbol{d}$ & $P$-value \\
\hline Total score & $53.22(7.53)$ & $50.68(7.89)$ & 0.329 & 0.034 \\
\hline Bedtime resistance & $11.16(2.15)$ & $10.39(2.23)$ & 0.351 & 0.024 \\
\hline Sleep onset delay & $6.34(1.24)$ & $6.18(1.39)$ & 0.121 & 0.417 \\
\hline Sleep anxiety & $6.36(2.20)$ & $5.76(1.90)$ & 0.291 & 0.065 \\
\hline Night waking & $4.41(1.67)$ & $3.92(1.23)$ & 0.334 & 0.033 \\
\hline Daytime sleepiness & $13.25(3.61)$ & $13.23(3.31)$ & 0.005 & 0.964 \\
\hline Parasomnias & $9.08(3.03)$ & $8.58(2.62)$ & 0.176 & 0.260 \\
\hline Sleep disordered breathing & $3.60(1.27)$ & $3.43(1.18)$ & 0.138 & 0.386 \\
\hline
\end{tabular}

Independent sample test was used.

Table II. Comparison of Kinder Lebensqualitätsfragebogen: Children's Quality of Life Questionnaire - revised (KINDL-R) scores between patient and control groups

\begin{tabular}{|lcccc|}
\hline KINDL-R & $\begin{array}{c}\text { Patients, } n=86 \\
\text { Mean (SD) }\end{array}$ & $\begin{array}{c}\text { Controls, } n=82 \\
\text { Mean (SD) }\end{array}$ & Cohen's $\boldsymbol{d}$ & $P$-value \\
\hline Total score & $85.41(15.69)$ & $89.97(13.00)$ & 0.316 & $\mathbf{0 . 0 4 3}$ \\
\hline Physical well-being & $13.54(3.75)$ & $15.45(2.97)$ & 0.564 & $\mathbf{0 . 0 0 0}$ \\
\hline Emotional well-being & $14.62(3.41)$ & $15.54(2.19)$ & 0.321 & $\mathbf{0 . 0 4 0}$ \\
\hline Self-esteem & $12.91(3.94)$ & $13.32(2.91)$ & 0.118 & 0.446 \\
\hline Family & $15.01(2.86)$ & $15.89(3.23)$ & 0.288 & 0.063 \\
\hline Social & $13.48(3.49)$ & $14.97(2.41)$ & 0.496 & $\mathbf{0 . 0 0 2}$ \\
\hline School & $12.17(3.26)$ & $13.90(2.38)$ & 0.255 & $\mathbf{0 . 0 2 1}$ \\
\hline
\end{tabular}

Independent sample test was used.

Table III. Correlations between Children's Sleep Habits Questionnaire (CSHQ) and Kinder Lebensqualitätsfragebogen: Children's Quality of Life Questionnaire - revised (KINDL-R) scores in the patient group

\begin{tabular}{|c|c|c|c|c|c|c|c|}
\hline $\begin{array}{l}\text { KINDL-R } \\
\text { CSHQ }\end{array}$ & $\begin{array}{l}\text { Total } \\
\text { score }\end{array}$ & $\begin{array}{c}\text { Physical } \\
\text { well-being }\end{array}$ & $\begin{array}{l}\text { Emotional } \\
\text { well-being }\end{array}$ & Self-esteem & Family & Social & School \\
\hline Total score & $-0.375^{\star *}$ & -0.190 & $-0.282^{\star *}$ & -0.200 & $-0.258^{\star}$ & $-0.279^{\star *}$ & -0.196 \\
\hline Bedtime resistance & $-0.215^{*}$ & -0.123 & -0.077 & -0.108 & $-0.242^{*}$ & -0.164 & -0.081 \\
\hline Sleep onset delay & $-0.236^{*}$ & $-0.233^{*}$ & -0.200 & $-0.234^{\star}$ & $-0.216^{\star}$ & $-0.231^{*}$ & $-0.254^{\star}$ \\
\hline Sleep anxiety & -0.032 & -0.027 & 0.069 & 0.104 & 0.064 & 0.052 & 0.102 \\
\hline Night waking & -0.168 & -0.032 & -0.105 & -0.060 & -0.166 & -0.021 & -0.010 \\
\hline Daytime sleepiness & $-0.221^{*}$ & -0.052 & -0.168 & -0.055 & -0.122 & $-0.226^{\star}$ & $-0.223^{\star}$ \\
\hline Parasomnias & $-0.267^{*}$ & $-0.231^{*}$ & -0.180 & -0.087 & -0.150 & -0.074 & -0.075 \\
\hline Sleep disordered breathing & -0.045 & -0.028 & -0.125 & 0.002 & -0.030 & 0.064 & 0.133 \\
\hline
\end{tabular}

Pearson's correlation analysis was used. ${ }^{*} P<0.05,{ }^{* *} p<0.01$.

esteem and family subscores were also lower in the patient group, but these were not statistically significant $(p>0.05)$.

The correlations between CSHQ and KINDL-R scores in the patient group are presented in Table III. As seen in the table, CSHQ total score $(r=-0.38$, $p=0.000)$, and bedtime resistance $(r=-0.22$, $p=0.047)$, sleep onset delay $(r=-0.24, p=0.029)$, daytime sleepiness $(r=-0.22, p=0.041)$, and parasomnias $(r=-0.27, p=0.013)$ subscores were negatively correlated with KINDL-R total score. On the other hand, KINDL-R total score $(r=-0.38$, 
Table IV. Hierarchical linear regression model evaluating predictors of health-related quality of life (HRQOL) in the patient group

\begin{tabular}{|c|c|c|c|c|}
\hline Step & $\beta$ & $R^{2}$ & $\Delta R^{2}$ & $\Delta F(\mathrm{~d} f)$ \\
\hline \multicolumn{5}{|l|}{ 1. Demographics } \\
\hline Age & -0.157 & 0.033 & - & - \\
\hline Female sex ${ }^{a}$ & -0.091 & - & - & - \\
\hline \multicolumn{5}{|l|}{ 2. Disease severity } \\
\hline Severe disease ${ }^{b}$ & $-1.142^{*}$ & $0.138^{*}$ & 0.105 & $1.113^{*}(1,82)$ \\
\hline 3. CSHQ & - & $0.250^{*}$ & 0.112 & $2.542^{*}(8,74)$ \\
\hline Total score & $-0.279^{*}$ & - & - & - \\
\hline Bedtime resistance & -0.197 & - & - & - \\
\hline Sleep onset delay & -0.224 & - & - & - \\
\hline Sleep anxiety & -0.207 & - & - & - \\
\hline Night waking & -0.035 & - & - & - \\
\hline Daytime sleepiness & -0.146 & - & - & - \\
\hline Parasomnias & -0.186 & - & - & - \\
\hline Sleep disordered breathing & -0.028 & - & - & - \\
\hline
\end{tabular}

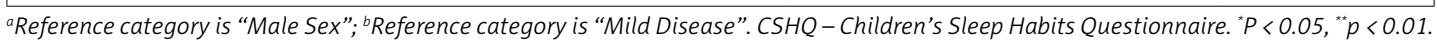

$p=0.000)$, and emotional well-being $(r=-0.28$ $p=0.009)$, family $(r=-0.26, p=0.016)$, and social $(r=-0.28, p=0.010)$ subscores were negatively correlated with CSHQ total score.

Table IV shows the hierarchical linear regression model evaluating the predictors of HRQOL (KINDL-R total score) in the patient group. The findings of step 1 showed that the variance accounted for by the demographic predictors (age and gender) equaled 0.03 (adjusted $R^{2}=0.01$ ), which was not significantly different from zero $(F=0.88, p=0.247)$. In the next step, the addition of the "disease severity" raised the variance of the model by 0.105 , which was a significant change $\left(\Delta \mathrm{F}_{[1,82]}=1.11, p=0.035\right)$. The final model, with the entry of all CSHQ scores, also raised the variance by 0.112 , which was again statistically significant $\left(\Delta \mathrm{F}_{[8,74]}=2.54, p=0.017\right)$.

The final model accounted for a total variance of 0.25 (adjusted $R^{2}=0.14$ ). In the final model, disease severity $(\beta=-1.14, p=0.024)$ and CSHQ total score $(\beta=-0.28, p=0.046)$ had significant negative associations with KINDL-R total score.

\section{Discussion}

One of the aims of this study was to examine the sleep problems and HRQOL in children and adolescents with SCD. In this context, this study compared sleep habits and behaviors and HRQOL between children and adolescents with SCD and typically developing healthy controls based on parent reports. Additionally, the study assess- ed the connection between sleep problems and HRQOL in SCD children to address a gap in the available literature. The study results are generally consistent with our hypothesis [18].

There are several studies on sleep problems among children and adolescents with SCD [7, 18-20]. These studies have generally suggested a high prevalence of sleep difficulties in SCD children. Valrie et al. [7] particularly reported more problems about reinitiating sleep after waking in the night, falling asleep, maintaining sleep, and going to bed. In another study, Hankins et al. [19] found an increased prevalence of obstructive symptoms, restless leg, sleep onset and maintenance insomnia. In the study of Daniel et al. [20], children with SCD experienced more enuresis, night wakings, and respiratory distress during sleep. Our findings are consistent with previous reports about the higher frequency of sleep disturbances in SCD and the kinds of sleep disturbances.

Many studies have detected low HRQOL among children and adolescents with SCD [1-3, 9, 21-23] Palermo et al. [10] found that SCD children had more restricted social, psychological, and physical well-being than healthy ones with similar socioeconomic characteristics. Sehlo and Kamfar [1] suggested significantly impaired HRQOL in all dimensions in children with SCD as compared to healthy controls. Beverung et al. [21] noted poor HRQOL in infants with SCD. Menezes et al. [3] reported that SCD was related to many limita- 
tions in different domains of HRQOL, especially school, social, emotional, and physical domains. Bhagat et al. [22] observed significantly lower overall HRQOL in children with SCD compared with other children with chronic illnesses such as diabetes mellitus, nephrotic syndrome, and congenital heart disease. A recent study conducted with SCD children showed that HRQOL was low in school, emotional, and physical domains as evaluated by adolescents, and was low in school and physical domains as evaluated by caregivers [2]. In the present study, emotional, physical, social, and school domains of HRQOL were significantly limited in children and adolescents with SCD as compared to healthy peers. Pain can be very common and highly destructive of physical and social activities in SCD children and can increase the risk of not attending school. Also, hospitalization may be necessary for the management of complications of SCD and may affect play activities and school attendance. Emotional problems may be associated with stigmatization in the community or chronic fatigue, which may prevent children from performing physical activities.

To the best of our knowledge, this is the first research to investigate the relationship between various types of sleep patterns and HRQOL in children with SCD. The study results showed that bedtime resistance, sleep onset delay, daytime sleepiness, and parasomnias were negatively associated with overall HRQOL. On the other hand, emotional well-being, family, and social dimensions of HRQOL were also negatively associated with overall sleep problems. These findings may indicate a bidirectional relationship, although the nature and direction of the relationship between sleep disorders and HRQOL in children and adolescents with SCD are undetermined. Sleep problems may cause poor HRQOL, and, vice versa, low HRQOL may lead to sleep disturbances. Future studies are warranted to reveal the pathophysiologic and behavioral mechanisms that may clarify the connection between sleep disorders and HRQOL in SCD children. Thus, precise targets for interventions (e.g. positive sleep hygiene) could be determined.

In the regression analyses predicting $\mathrm{HRQOL}$, disease severity and sleep problems were significant predictors. Specifically, sleep problems were found to independently predict overall $\mathrm{HRQOL}$ in this population, even after controlling for demographic factors and disease severity. Insufficient sleep has been shown to relate to lower academic performance and increased psychiatric and behavioral problems in healthy children [13, 24]. In the context of SCD, poor sleep may be related to more important results for children and adolescents. The data of the present study may support the idea that in children and adolescents with SCD, sleep problems are associated with broad impairments in daily functioning and overall HRQOL.

There are some limitations to consider when evaluating the results of this study. A major limitation is reliance on parent reports. The assessment of sleep disturbances and HRQOL was made by parent reports only. Parental mental and emotional problems, if present, may bias their perception of child sleep quality and HRQOL. Polysomnography and actigraphy are more reliable methods and may be performed to study sleep disturbances in children more precisely. A second limitation is due to not evaluating the effects of sociodemographic factors and SCD phenotypes. These factors may have an impact on clinical manifestation, sleep problems, and quality of life. Furthermore, the use of a cross-sectional study design eliminates the ability to make causal attributions. To truly learn the complex association between sleep problems and HRQOL in children and adolescents with SCD, longitudinal studies are needed.

Consequently, the study results indicate that sleep disturbances in children and adolescents with SCD may be a significant predictor of overall HRQOL. For those with SCD, sleep quality should be evaluated with parent- and clinician-rated assessment tools regularly. This evaluation can be very significant in terms of several factors. The diagnosis and efficient handling of sleep disorders may lead to amelioration in HRQOL and increase the compliance of children with medical interventions, school attendance, and daily activities.

\section{Conflict of interest}

The authors declare no conflict of interest.

\section{References}

1. Sehlo MG, Kamfar HZ. Depression and quality of life in children with sickle cell disease: the effect of social support. BMC Psychiatry 2015; 15: 78.

2. Kambasu DM, Rujumba J, Lekuya HM, et al. Healthrelated quality of life of adolescents with sickle cell disease in sub-Saharan Africa: a cross-sectional study. BMC Hematol 2019; 19: 9.

3. Menezes AS de O da P, Len CA, Hilário MOE, et al. Quality of life in patients with sickle cell disease. Rev Paul Pediatr 2013; 31: 24-9.

4. Gunes S, Ekinci O, Feyzioglu A, et al. Sleep problems in children with autism spectrum disorder: clinical correlates and the impact of attention deficit hyperactivity disorder. Neuropsychiatr Dis Treat 2019; 15: 763-71.

5. Shapiro BS, Dinges DF, Orne EC, et al. Home management of sickle cell-related pain in children and adolescents: natural history and impact on school attendance. Pain 1995; 61: 139-44.

6. Lewin DS, Dahl RE. Importance of sleep in the management of pediatric pain. J Dev Behav Pediatr 1999; 20: 244-52. 
7. Valrie CR, Gil KM, Redding-Lallinger $R$, et al. The influence of pain and stress on sleep in children with sickle cell disease. Child Health Care 2007; 36: 335-53.

8. Güneş S, Ekinci Ö. Health-related quality of life in children and adolescent with ASD: the role of mothers' emotional symptoms. Anatol J Psychiatry 2019; 20: 548-55.

9. Polikandrioti M, Panoutsopoulos G, Tsami A. Assessment of quality of life and anxiety in heart failure outpatients. Arch Med Sci Atheroscler Dis 2019; 4: 38-46.

10. Palermo TM, Drotar D, McGowan K. Parental report of health-related quality of life in children with sickle cell disease. J Behav Med 2002; 25: 269-83.

11. Franzen MD. The Wechsler Intelligence Scales for Children - the WISC-R, WISC-III, and WPPSI-R. In: Reliability and Validity in Neuropsychological Assessment. Critical Issues in Neuropsychology. Springer, Boston 2000; 71-89.

12. Ekinci O, Okuyaz Ç, Gunes S, et al. Sleep problems in pediatric epilepsy and ADHD: the impact of comorbidity. Epilepsy Behav 2017; 71: 7-12.

13. Güneş S. Sleep problems in children and adolescents with $\beta$-thalassemia major. Cukurova Med J 2019; 44: 1267-71.

14. Fiş NP, Arman A, Ay P, et al. The validity and the reliability of Turkish version of Children's Sleep Habits Questionnaire. Anatol J Psychiatry 2010; 11: 151-60.

15. Ravens-Sieberer U, Bullinger M. Assessing health-related quality of life in chronically ill children with the German KINDL: first psychometric and content analytical results. Qual Life Res 1998; 7: 399-407.

16. Eser E, Yüksel H, Baydur $H$, et al. The psychometric properties of the new Turkish generic health-related quality of life questionnaire for children (Kid-KINDL). Turk Psikiyatri Derg 2008; 19: 409-17.

17. Goldstein-Leever A, Peugh JL, Quinn CT, et al. Disease self-efficacy and health-related quality of life in adolescents with sickle cell disease. J Pediatr Hematol Oncol 2020; 42: 141-4.

18. Downes M, de Haan M, Kirkham FJ, et al. Parent reported sleep problems in preschool children with sickle cell anemia and controls in East London. Pediatr Blood Cancer 2017; 64. DOI: 10.1002/pbc.26337.

19. Hankins JS, Verevkina NI, Smeltzer MP, et al. Assessment of sleep-related disorders in children with sickle cell disease. Hemoglobin 2014; 38: 244-51.

20. Daniel LC, Grant M, Kothare SV, et al. Sleep patterns in pediatric sickle cell disease. Pediatr Blood Cancer 2010; 55: 501-7.

21. Beverung LM, Bemrich-Stolz C, Torres S, et al. Healthrelated quality of life in infants with sickle cell disease. J Pediatr Hematol Oncol 2015; 37: 590-4.

22. Bhagat VM, Baviskar SR, Mudey AB, et al. Poor health related quality of life among patients of sickle cell disease. Indian J Palliat Care 2014; 20: 107-11.

23. Wrotniak BH, Schall JI, Brault ME, et al. Health-related quality of life in children with sickle cell disease using the child health questionnaire. J Pediatr Health Care 2014; 28: 14-22.

24. Wolfson AR, Carskadon MA. Sleep schedules and daytime functioning in adolescents. Child Dev 1998; 69: 875-87. 